\title{
Funcionamiento familiar en el riesgo y la protección de trastornos del comportamiento alimentario*
}

Family Functioning in the Risk and Protection of the Eating Disorders

Recibido: abril 20 de 2009 | Revisado: septiembre 14 de 2009 | Aceptado: noviembre 10 de 2009

\author{
Ana Olivia Ruiz-Martínez ** \\ Rosalía VÁzQUEZ-ARÉvalo \\ JuAN MANUEl MANCILla-DíaZ \\ XOCHITL LÓPEZ-AGUILAR \\ GEORGINA L. ÁlVAREZ-RAYÓN
}

Universidad Nacional Autónoma de México, FES, Iztacala

AntONIO TENA-SUCK ${ }^{* * * *}$

Universidad Iberoamericana, Fundación CBA, México

Para citar este artículo. Ruíz-Martínez, A.O., Vásquez-Arévalo, R., Mancilla-Díaz, J.M., López-Aguilar, X., Álvarez-Rayón, G., \& Tena-Suck, A. (2010). Funcionamiento familiar en el riesgo y la protección de trastornos del comportamiento alimentario. Universitas Psychologica, 9 (2), 447-455.

* Trabajo de investigación apoyado por PAPIIT IN304909 y CONACyT 50305-H.

**Ana Olivia Ruíz Martínez (ana_olivi@yahoo.com. mx), Rosalía Vázquez Arévalo (rvamap@unam. mx), Juan Manuel Mancilla Díaz (jmmd@unam. mx), Xochitl López Aguilar (xochitll@unam.mx), Georgina L. Alvarez Rayón (alvarezr@unam.mx). Universidad Nacional Autónoma de México-FES Iztacala. División de Investigación y Posgrado. Proyecto de Nutrición. Av. de los Barrios \# 1, Los Reyes Iztacala. Estado de México, México. C.P. 54090. Tel. 56231333 exts. \# 39736, 39737, 39738. Fax. 53 907604

**** Antonio Tena Suck (antonio.tena@uia.mx) Universidad Iberoamericana, Ciudad de México, Prolongación Paseo de la Reforma 880. Lomas de Santa Fe, México.

C.P.01219 Distrito Federal. Tel. 52 (55) 59504046.

\section{RESUMEN}

El objetivo del presente estudio fue analizar los aspectos del funcionamiento familiar que explican parte del riesgo y la protección de los Trastornos del Comportamiento Alimentario (TCA): Anorexia Nerviosa (AN), Bulimia Nerviosa (BN) y Trastornos del Comportamiento Alimentario No Especificados (TCANE). Participaron 70 mujeres con TCA (16 con AN, 24 con $\mathrm{BN}$, y 30 TCANE) y 30 mujeres sin TCA, con un promedio de 18 años. Todas fueron entrevistadas para su diagnóstico y además contestaron a 3 cuestionarios: FES de ambiente familiar; EAT-40; y BULIT, para evaluar sintomatología. Los resultados señalan que para AN, BN y TCANE la expresión fue factor de riesgo y las actividades intelectual-culturales de protección, pero en caso de los TCA específicos se suman la organización como factor de riesgo y la actuación como factor protector. Los datos indican diferencias en la percepción del grado de afectación familiar entre los diferentes tipos de TCA.

Palabras clave autores

Trastornos del comportamiento alimentario, familia, funcionamiento familiar. Palabras clave descriptores

Trastornos alimenticios, anorexia nerviosa, bulimia nerviosa, salud de la familia.

\footnotetext{
A B S T R A C T

Objective: We analyzed the elements of the family functioning that explain the risk and protection of the ED: Anorexia Nervosa (AN), Bulimia Nervosa (BN) and Eating Disorder Non-Specific (EDNOS). Method: Seventy patients with ED (16 AN, 24 BN and 30 EDNOS) and 30 female students were interviewed and completed three questionnaires: FES, EAT and BULIT. Results: The ED patients (AN, BN and EDNOS) perceived the expression was a risk factor and the intellectual-cultural activities were protection factor. In the group of ED Specific (AN and BN) the organization was a risk factor and actuation was protector factor. Conclusion: The results confirmed differences in the family functioning between types of ED Specific and Non-Specific.

Key words authors

Eating Disorders, Family, Family Functioning.

Key words plus

Eating Disorders, Anorexia Nervosa, Bulimia Nervosa, Family Health.
} 


\section{Introducción}

Las Trastornos del Comportamiento Alimentario (TCA) atraen el interés de los profesionales de la salud dado los problemas psicológicos, el deterioro de la salud lo difícil del tratamiento y la alta tasa de recaídas, que afectan a quienes los padecen.

Los TCA son aquellos procesos psicopatológicos que conllevan graves anormalidades en las actitudes y comportamientos respecto a la ingestión de alimentos, su base se encuentra en una alteración psicológica que se acompaña de una distorsión en la percepción corporal y de un miedo intenso a la obesidad. Comprenden de acuerdo al DSM-IV-TR (Diagnostic and Statistical Manual of Mental Disorders, Text Revision, APA, 2000) a la Anorexia Nerviosa (AN), la Bulimia Nerviosa (BN) y los Trastornos de la Comportamiento Alimentarios No Especificados (TCANE), también conocidos como Síndromes Parciales o Trastornos Subclínicos.

La psicopatología alimentaria se caracteriza por la preocupación excesiva por el peso y la figura, la motivación para adelgazar, así como, sentimientos negativos, obsesiones y compulsiones con respecto a la alimentación. De manera particular, la Anorexia Nerviosa (AN) se define por las conductas de restricción alimentaria y emaciación que se observan en el bajo peso y la amenorrea, en tanto que en la Bulimia Nerviosa (BN) se presentan atracones seguidos de conductas compensatorias, ya sean éstas purgativas o no purgativas.

La complejidad de estos trastornos requiere ser abordada desde una perspectiva multifactorial, donde los factores familiares resultan relevantes al ser el primer grupo que trasmite creencias, actitudes y modela conductas relacionadas a los alimentos que acompañarán al individuo, a lo largo de su vida.

Se considera que la familia puede actuar como un factor etiológico, debido a que su funcionamiento puede propiciar la aparición de tales trastornos (Williamson, 1990); o como un factor mantenedor, en cuanto que las interrelaciones del entorno familiar permiten que los síntomas permanezcan a través del tiempo (Treasure et al., 2007), y más recientemente se ha puesto hincapié en considerarla en su papel protector (Fonseca, Ireland \& Resnick, 2002), donde sus características proveen a sus integrantes de habilidades para contrarrestar o evitar los factores de riesgo. De tal forma que un contexto familiar positivo, se asocia con menor vulnerabilidad y mayor protección social, mientras que un contexto negativo presupone pocas destrezas y mayor vulnerabilidad (Davis, Shuster, Blackmore \& Fox, 2004).

$\mathrm{Al}$ estudiar el funcionamiento familiar, entendido como las interrelaciones que establecen los integrantes del grupo familiar, se han encontrado diferencias entre las familias con y sin TCA; las familias afectadas muestran menor: cohesión, flexibilidad (Vázquez, Álvarez, Mancilla \& Raich, 2000; Vidovic, Juresa, Begovaci, Mahnik \& Tocilj, 2005), expresión emocional y comunicación intrafamiliar (Sánchez, Serna, Seaona \& Páramo, 2003), así como, mayor rechazo y sobreprotección paterna (Jones, Leung \& Harris, 2006).

Asimismo, se observa que las hijas con TCA perciben que en su familia se le da menos importancia a la dimensión de desarrollo que comprende características tales como: autonomía, actividades sociales y actividades culturales (Vázquez, Raich, Viladrich, Álvarez \& Mancilla, 2001). En suma, las personas con TCA perciben un ambiente familiar significativamente más deteriorado y sentimientos negativos hacia sus familiares; incluso algunas pacientes refieren sentirse ignoradas o no amadas por sus madres (Beato, Rodríguez, Belmonte \& Martínez, 2004).

De manera particular, se ha distinguido el funcionamiento familiar de acuerdo al tipo de trastorno que las aqueja. Las pacientes con AN señalan mayor insatisfacción con su vida familiar, rigidez (Cook-Darzens, Doyen, Falissard \& Mouren, 2005) dificultad en la solución de problemas y altos niveles de crítica y hostilidad (Emmanuelli et al., 2004; Kyriacou, Treasure \& Schmidt, 2008).

De igual manera, las hijas con AN perciben que sus familias son altamente cohesivas, lo que podría perjudicar la individualidad de sus miembros, favoreciendo conductas de control y sobreprotección (Vázquez et al. 2001). No obstante, en estudios 
recientes no se ha corroborado esta tendencia hacia la sobreprotección, sino que se documenta baja cohesión familiar al contemplar la percepción de hijas y/o padres (Cook-Darzens et al., 2005; Tereno, Soares, Martins, Celani \& Sampaio, 2008).

Por su parte, las familias de las personas con BN presentan actitudes de hostilidad y negativismo frente a las necesidades emocionales de las hijas, de manera que éstas perciben mayor desintegración y menor apoyo e independencia (Shisslak, Mckeon \& Crago, 1990). Las personas afectadas por $\mathrm{BN}$ perciben que en su ambiente familiar existen bajos niveles de expresión y alto conflicto (Okon, Greene \& Ellen, 2003). También se ha documentado que estas pacientes presentan altos niveles de emoción expresada (intrusividad, repuesta emotiva, actitudes negativas y poca tolerancia) haciendo difícil su cuidado por parte de familiares y cuidadores (Winn et al., 2007).

Como se aprecia, ya se cuenta con descripciones del funcionamiento familiar de los TCA específicos (AN y BN), en México hace falta confirmar estos hallazgos y al igual que a nivel internacional aportar datos al respecto de personas con TCANE, clasificación que incluye a aquellos TCA que no cumplen con todos los criterios de AN o BN (APA, 2000); su frecuencia oscila entre $2.37 \%$ y $3.80 \%$ superando la prevalencia de AN $(0.50 \%$ a $1 \%)$ y BN (1\% y el 3\%) (Machado, Machado, Goncalves \& Hoek, 2007; Vázquez, López, Álvarez, Franco \& Mancilla, 2004) y cuya relevancia se ve reflejada en los múltiples intentos de identificar los subgrupos que la componen, entre los que destacan diferentes tipos de pacientes: restrictivos, purgativos, normopesos con atracones y preocupación por la figura, normopesos con vómito y abuso de laxantes, sobrepeso u obesidad en ausencia de psicopatología alimentaria y el Trastorno por Atracón (TPA) (Mitchell et al., 2007; Wade, 2007).

Con la intención de aportar evidencia de la manera en que la familia está incidiendo en la presencia de psicopatología alimentaria, el propósito del presente estudio fue analizar algunos aspectos del funcionamiento familiar que explican el riesgo y la protección de los Trastornos del Comportamiento Alimentario (TCA): Anorexia Nerviosa
(AN), Bulimia Nerviosa (BN) y Trastornos del Comportamiento Alimentario No Especificados (TCANE).

\section{Método}

La presente investigación fue no experimental con temporalidad transversal y de alcance correlacional.

\section{Participantes}

Participaron 100 mujeres de 11 a 29 años $(\bar{X}=$ 18.2, $D E=3.86)$, habitantes del área metropolitana de la Ciudad de México, que fueron divididas en dos grupos: con TCA y sin TCA (control), de acuerdo con el diagnóstico establecido mediante una entrevista clínica individual para tal fin.

El grupo con TCA se conformó con 70 mujeres que solicitaron servicio en una clínica privada a quienes se les diagnosticó mediante una entrevista individual basada en los criterios del DSM-IV-TR (APA, 2000), confirmando la existencia de 16 personas con $\mathrm{AN}, 24$ con $\mathrm{BN}$, y 30 con TCANE (Tabla 1).

TABLA 1

Características de las participantes

\begin{tabular}{|c|c|c|}
\hline $\begin{array}{c}\text { Tipo de Trastorno } \\
\text { del Comportamiento } \\
\text { Alimentario }\end{array}$ & $\begin{array}{l}\text { Proce- } \\
\text { dencia }\end{array}$ & $\begin{array}{l}\text { Promedio de } \\
\text { edad en años }\end{array}$ \\
\hline \multirow{2}{*}{$\begin{array}{l}\text { Anorexia Nerviosa } \\
(n=16)\end{array}$} & CBA 13 & \multirow{2}{*}{$\bar{\chi}=18.63$} \\
\hline & PIN 3 & \\
\hline $\begin{array}{l}\text { Bulimia Nerviosa } \\
(n=24)\end{array}$ & CBA 24 & $\bar{\chi}=19.79$ \\
\hline \multirow{2}{*}{$\begin{array}{l}\text { Trastorno del Comportamien- } \\
\text { to Alimentario No Específico } \\
(n=30)\end{array}$} & CBA 9 & \multirow{2}{*}{$\bar{X}=17.90$} \\
\hline & PIN 21 & \\
\hline $\begin{array}{l}\text { Control } \\
(\mathrm{n}=30)\end{array}$ & PIN 30 & $\bar{\chi}=18.24$ \\
\hline
\end{tabular}

PIN: Proyecto de Investigación en Nutrición, UNAM-FES Iztacala.

CBA: Fundación contra la Bulimia y Anorexia Nerviosas, A.C.

Fuente: elaboración propia. 
El grupo sin TCA, que actúo como grupo control, estuvo formado por 30 mujeres pertenecientes a la población comunitaria, específicamente estudiantes de nivel medio superior y superior, a quienes también se les realizó la entrevista diagnóstica para confirmar la ausencia de TCA.

\section{Instrumentos}

Test de actitudes alimentarias (EAT-40) de Garner y Garfinkel (1979).

Evalúa la presencia de síntomas relacionados con los Trastornos del Comportamiento Alimentario, su validación para México (Álvarez, Mancilla, Vázquez, Unikel, Caballero \& Mercado, 2004) contempla 5 factores: 1) Dieta restrictiva, 2) Bulimia, 3) Motivación para adelgazar, 4) Preocupación por la comida y 5) Presión social percibida. Considerando punto de corte $\geq 28$ para mujeres mexicanas, con un alpha de Cronbach de 0.90.

Cuestionario de Bulimia (BULIT) de Smith y Telen (1984).

Evalúa la presencia de síntomas y preocupaciones característicos de la Bulimia Nerviosa; en su validación para México (Álvarez, Mancilla \& Vázquez, 2000) se obtuvo una estructura de 3 factores: 1) Sobreingesta y sensación de pérdida de control sobre la alimentación, 2) Sentimientos negativos posteriores a la sobreingesta, y 3) Conductas compensatorias. El punto de corte más adecuado para mujeres mexicanas fue $\geq 85$, con un alpha de Cronbach de 0.88 .

Entrevista para el Diagnóstico de Trastorno Alimentario (IDED) / Interview for the Diagnosis of Eating Disorders de Kustlesic Williamson, Gleaves, Barbin y Murphy- Eberenz (1998).

Tiene por objetivo detectar conductas relacionadas con los TCA: AN, BN, TCANE, considerando los criterios diagnósticos del DSM-IV-TR (APA, 2000). La validez de contenido contempló a diez expertos que la calificaron favorablemente. Es decir, que los especialistas juzgaron que evaluaba todos los aspectos necesarios para formular el diagnóstico de un TCA y diferenciar entre los tipos y subtipos. En cuanto a la validez concurrente, el IDED correlacionó con el factor dieta del EAT ( $r$ $=.60)$, atracón y vómito del BULIT $(r=.68$ en ambos casos). Formato adaptado para la población mexicana por el Proyecto de Investigación en $\mathrm{Nu}$ trición de la UNAM-FES, Iztacala.

Escala de Clima Social en la Familia (FES) de Moos y Moos (1980), validado en España por Seisdedos, Cruz y Cordero (1984).

Esta escala muestra las características socioambientales de la familia, evalúa y describe las relaciones interpersonales entre los miembros de la familia, los aspectos de desarrollo que tienen mayor importancia en ella y su estructura básica. Contiene 90 elementos, agrupados en 10 subescalas (cohesión, expresión, conflicto, autonomía, actuación, intelectual-cultural, social-recreativa y moralidad-religiosidad, organización y control). En México, se obtuvo un alfa total de Cronbach de .78 y el análisis factorial encontró los mismos factores que la escala original (Ayala, Fulgencio, Chaparro \& Pedroza, 2000).

\section{Procedimiento}

En primer lugar se informó a todas las participantes sobre el propósito del estudio, se solicitó su participación voluntaria, garantizándoles la confidencialidad de la información y se obtuvo el consentimiento informado. Todas las participantes fueron diagnosticadas mediante la aplicación de la entrevista IDED, realizada por especialistas en TCA, para ser asignadas a un grupo.

En el caso de la muestra clínica, a las pacientes se les aplicaron individualmente los cuestionarios -EAT, BULIT y FES-, al momento de solicitar tratamiento y se les diagnosticó mediante entrevista. Posteriormente fueron atendidas.

Para la muestra comunitaria, la aplicación de los cuestionarios fue grupal; posteriormente, se entrevistó a aquellas personas que no rebasaron el punto 
de corte de los instrumentos de tamizaje (EAT $\geq 28$ y BULIT $\geq 85$ ) y se les aplicó la entrevista diagnóstica a fin de corroborar la ausencia de TCA.

\section{Análisis de resultados}

Los datos recabados se analizaron mediante correlaciones de Pearson entre el FES, EAT y BULIT; evaluando así, las asociaciones entre la percepción del funcionamiento familiar y los síntomas de TCA.

Para someter a prueba si las subescalas que habían correlacionado con los síntomas de TCA podían actuar como factores explicativos de la presencia de TCA y evaluar su efecto sobre los TCA específicos y nos específicos, se realizaron regresiones logísticas dicotómicas generando dos modelos explicativos:

I. TCA específicos (AN y BN) vs. Control

II. TCANE vs. Control.

En las regresiones logísticas se empleó el método paso a paso, y se probaron diferentes modelos para asegurar la máxima bondad de ajuste; así como, un adecuado porcentaje de sujetos correctamente clasificados. Para cada uno de los predictores, se calcularon los coeficientes y sus errores estándar. Las variables fueron eliminadas del modelo, basándose en la prueba de razón likelihood (-2LL). La calidad del ajuste fue evaluada por la ji cuadrada dada en las regresiones. La ausencia de TCA fue codificada con 0 y la presencia de TCA fue codificada con 1 , quedando así determinado el grupo control de referencia.

\section{Resultados}

En el análisis correlacional se subdividió el grupo con TCA de acuerdo al diagnóstico de las participantes, para observar cuáles asociaciones ocurrían en cada tipo de trastorno. En la Tabla 2 se aprecia un resumen de las correlaciones significativas y con un coeficiente mayor a .30. En el grupo de AN sólo existe una correlación significativa y negativa entre el total del BULIT y la subescala Expresión, por lo que al parecer a mayor sintomatología de bulimia menor expresividad. No se encontró nin- guna asociación significativa en el grupo de BN. Para los pacientes de TCANE resultó significativa la asociación negativa entre el total del EAT y Cohesión, así como, entre el total del BULIT y Conflicto, por lo que a mayor sintomatología de TCA menor cohesión y conflicto. El grupo control también mostró asociación entre el total del EAT y Expresión, refiriendo que a menor sintomatología de TCA mayor expresión.

\section{TABLA 2}

Resumen de las correlaciones de Pearson entre percepción del funcionamiento familiar y síntomas de TCA, que fueron significativas y con $r h o \geq 30$

\begin{tabular}{|c|c|c|c|c|}
\hline \multicolumn{5}{|c|}{ Subescalas del FES } \\
\hline $\begin{array}{l}\text { Grupos por } \\
\text { Diagnóstico }\end{array}$ & & $\begin{array}{l}\text { Cohe- } \\
\text { sión }\end{array}$ & $\begin{array}{l}\text { Expre- } \\
\text { sión }\end{array}$ & $\begin{array}{l}\text { Conflic- } \\
\text { to }\end{array}$ \\
\hline \multirow{2}{*}{$\begin{array}{l}\text { Anorexia } \\
(\mathrm{n}=16)\end{array}$} & EAT & - & -.33 & - \\
\hline & BULIT & - & $-.57 *$ & .43 \\
\hline \multirow{2}{*}{$\begin{array}{l}\text { Bulimia } \\
(\mathrm{n}=24)\end{array}$} & EAT & - & - & .31 \\
\hline & BULIT & -.38 & - & .34 \\
\hline \multirow{2}{*}{$\begin{array}{l}\text { TCANE } \\
(\mathrm{n}=30)\end{array}$} & EAT & $-.37 *$ & - & - \\
\hline & BULIT & - & - & $-.44 *$ \\
\hline \multirow{2}{*}{$\begin{array}{l}\text { CONTROL } \\
(\mathrm{n}=30)\end{array}$} & EAT & -.35 & $.47^{* *}$ & - \\
\hline & BULIT & - & -.31 & - \\
\hline
\end{tabular}

$* p<0.05 y * p<0.01$.

Fuente: elaboración propia.

Posteriormente, se realizó el análisis multivariado mediante regresiones logísticas, utilizando las subescalas del funcionamiento familiar como variables predictoras y como variables predecibles: a) presencia de TCA (AN y BN) vs. Control, y b) presencia de TCANE vs. Control.

El primer modelo indicó los factores explicativos para la presencia de los TCA específicos (AN y BN), encontrando que Expresión y Organización actuaron como factores de riesgo, en cambio Actuación e Intelectual-Cultural mostraron un efecto protector (véase Tabla 3). Contando con una adecuada bondad de ajuste $\left(R^{2}=0.54,-2 \mathrm{LL}=\right.$ $\left.60.02, \mathrm{Ji}^{2}=35.58, \mathrm{Sig} .=.001\right)$, y clasificando correctamente al $80 \%$ las participantes. 
El segundo modelo, señaló a la Expresión como factor de riesgo para los casos de TCANE y como factor protector las actividades Intelectuales-Culturales. En la Tabla 4, se aprecian los estadísticos de la bondad de ajuste del modelo $\left(R^{2}=0.35,-2 L L=\right.$ $64.90, \mathrm{Ji}^{2}=18.27$, Sig. $\left.=.0001\right)$, el cual clasificó correctamente al $70 \%$ de las participantes.

\section{Discusión}

La propuesta de este estudio fue indagar los aspectos del funcionamiento familiar que explican los factores de riesgo y protección de las familias con un miembro con TCA.

Como primera parte del análisis, se realizaron correlaciones entre los cuestionarios de sintomatología y el de familia, dividiendo a la muestra de TCA en: AN, BN y TCANE. Estos resultados brindaron una primera aproximación al análisis de las variables que se someterían al modelo de regresión logística, pero además aportaron información sobre las asociaciones de las participantes a los cuestionaros de sintomatología (EAT y BULIT) y el de Familia (FES).
En este sentido, en el grupo de AN se observó que a mayores síntomas bulímicos menor expresión, lo que concuerda con Emmanuelli et al. (2004) quienes advierten sobre la dificultad de la comunicación que presentan dichas familias.

Sorprendentemente en el grupo con BN, no se encontraron asociaciones significativas con ninguna de las subescalas del funcionamiento familiar, lo cual no era esperado ya que la literatura señala asociaciones entre la $\mathrm{BN}$ y mayor conflicto, menor expresividad emocional, cohesión y carencia de soporte social (Shisslak, Mckeon \& Crago, 1990). Esto muestra divergencias en la manera de responder a los cuestionarios de sintomatología (EAT y BULIT), y merece ser analizado en estudios posteriores porque sabemos que las participantes contaban con el TCA. En cuanto al grupo de TCA$\mathrm{NE}$, las participantes de este grupo percibieron a sus familias con menor integración, más distantes y que no se apoyan entre sí e, inesperadamente, ello trae una menor posibilidad de situaciones problemáticas o conflictos.

Estas relaciones entre sintomatología de TCA y funcionamiento familiar, coincide con la litera-

TABLA 3

Regresión logística de factores de riesgo y protección de los TCA específicos: AN y BN

\begin{tabular}{lccccccc}
\hline \multirow{2}{*}{ Variables } & B & EE & Wald & Sig. & Exp (B) & \multicolumn{2}{c}{ Intervalo de confianza } \\
\hline Expresión & -.84 & .22 & 14.56 & .001 & 0.43 & 0.28 & 0.67 \\
Actuación & .54 & .22 & 5.72 & .017 & 1.73 & 1.10 & 2.71 \\
Intelectual & .48 & .22 & 4.75 & .029 & 1.62 & 1.05 & 2.50 \\
Organización & -.47 & .18 & 6.65 & .010 & 0.62 & 0.44 & 0.89 \\
\hline
\end{tabular}

Bondad de ajuste $\left(R^{2}=0.54,-2 L L=60.02, \mathrm{Ji}^{2}=35.58\right.$, Sig. $\left.=.001\right)$.

Fuente: elaboración propia.

TABLA 4

Regresión logística de factores de riego y protección de los TCANE

\begin{tabular}{lccccccc}
\hline \multirow{2}{*}{ Variables } & B & EE & Wald & Sig. & Exp (B) & \multicolumn{2}{c}{ Intervalo de Confianza } \\
\cline { 6 - 8 } & & & & & & Inferior & Superior \\
\hline Expresión & -.70 & 0.21 & 11.70 & 0.001 & 0.50 & 0.33 & 0.74 \\
Intelectual & .48 & 0.19 & 6.03 & 0.01 & 1.61 & 1.10 & 2.36 \\
\hline
\end{tabular}

Bondad de ajuste del modelo $\left(R^{2}=0.35,-2 \mathrm{LL}=64.90, \mathrm{Ji}^{2}=18.27\right.$, Sig. $\left.=.0001\right)$.

Fuente: elaboración propia. 
tura, al señalar que la psicopatología alimentaria se asocia con la presencia de ambientes familiares desfavorecedores para el desarrollo óptimo del individuo (Davis, Shuster, Blackmore \& Fox, 2004). No obstante, la diferencias entre los grupos indican nuevamente la necesidad de ampliar las muestras, para poder dividir los grupos de acuerdo a los tipos y subtipos de TCA (Emmanuelli et al., 2004; Vidovic' et al., 2005).

Ahora bien, al averiguar cuáles de los factores del funcionamiento familiar podrían actuar como predictores y protectores, por el número de participantes se realizó de manera independiente con los TCA específicos (AN y BN) y los TCA no Específicos (TCANE).

Se encontró que la expresión en todos los grupos actuó como el factor más importante que explicó el riesgo de TCA, al ser la variable que explica mayor cantidad de varianza; lo que sugiere que al dejar de compartir emociones y sentimientos dentro de estas familias, se dejan al descubierto necesidades básicas de aceptación y afecto como lo han encontrado Sánchez et al. (2003). Por su parte, la variable denominada intelectual-cultural coincidió en ser la variable de protección para todos los grupos de TCA, ésta refiere al interés que se pone en actividades políticas, sociales, intelectuales y culturales; lo cual resulta coincidente a lo señalado por Vázquez et al. (2001) quienes encontraron que las hijas con TCA consideraban que sus familias no mostraban interés en este tipo de actividades.

Sólo para el caso de las participantes con AN y BN, la variable organización explicó el riesgo y la variable actuación fue protectora de los TCA -con menor potencia estadística que las anteriores variables-. La organización se entiende como la falta de planificación en las actividades cotidianas y la carencia de asignación de las responsabilidades familiares, es un rasgo más asociado a los pacientes con BN (Garner, Garfinkel \& O'Shaugnessy, 1985; Strober \& Humphrey, 1987), y siendo la mayor parte de este grupo pacientes con $\mathrm{BN}$ podría entenderse este resultado.

Como contraparte protectora, aparece la variable actuación que representa el grado en que las actividades de la escuela o el trabajo se enmarcan en la acción competitiva, es una característica que había sido referida en la literatura como orientación al logro, y más asociado a familias con AN (Vázquez, 2001), no parece una característica positiva en el ámbito familiar, por lo que será necesario seguir estudiándola para conocer en qué sentido está su papel en la protección, quizás se enmarca dentro la línea de fomentar actividades extrafamiliares, ya que otro factor protector fue mayor cantidad de actividades intelectuales y culturales, lo cual quizá brinda una oportunidad a mejorar la organización familiar.

Llama la atención que algunas de las variables que presentaron asociaciones con la sintomatología de TCA y que además son frecuentemente citadas en la literatura, no aparecieran como factores predictores, tal es el caso de la cohesión y el conflicto. Con referencia a la cohesión, Huon et al. (2002) encontraron que esta parte del funcionamiento familiar actuaba como predictora de la dieta -considerada una conducta asociada a la psicopatología alimentaria-, mientras que Houston y Cashwell (2000) reafirmaron su efecto predictor sobre las conductas de TCA; no obstante, ambos estudios se realizaron con población estudiantil que no presentaba trastorno. Esta situación nos lleva a aseverar que los factores predictivos sobre conductas de riesgo en población comunitaria, son diferentes a los que actúan en personas que ya tienen diagnosticado un TCA, reiterando así lo oportuno del análisis en muestras clínicas.

En cuanto al conflicto, Vázquez et al. (1998) ya habían reportado que en su muestra clínica no se presentaban puntuaciones altas de conflicto. En la presente investigación, al observar los resultados del análisis por diagnóstico, también se observa que esta escala sólo se asoció con la sintomatología de TCA en el subgrupo de TCANES, población que no fue considerada en el análisis de los autores anteriormente citados.

Por último, es importante reconocer que este estudio toma en cuenta únicamente la percepción de las hijas sobre el funcionamiento familiar, por lo que ofrece un enfoque unilateral. Tal como lo señalan Dancyger, Fornari, Scionti, Wisosky y Sunday (2005), es importante comparar la percep- 
ción del funcionamiento familiar entre diferentes miembros de la familia, porque existen diferencias importantes entre la percepción de hijas y padres; por lo que en futuros estudios habrá que agregar la perspectiva paterna, a fin de evaluar si estas relaciones entre el funcionamiento familiar y los TCA se mantienen o si se presentan variaciones de acuerdo con diferentes integrantes de su familia.

\section{Referencias}

Álvarez, G. L., Mancilla J. M. \& Vázquez, R. (2000). Propiedades psicométricas del test de bulimia (BULIT). Psicología Contemporánea, 7(1), 74-85.

Álvarez, G. L., Mancilla, J. M., Vázquez, R., Unikel, S. C., Caballero, R. A. \& Mercado, C. D. (2004). Validity of the Eating Attitudes Test: A study of Mexican eating disorders patients. Eating Weight Disorders, 9(4), 243-248.

American Psychiatric Association. (2000). Diagnostic and Statistical Manual of Mental Disorders. DSMIV-TR. Washington DC: Autor.

Ayala, V. H., Fulgencio, J. M., Chaparro, C. A \& Pedroza, C. F. (2000). Resultados preliminaries del proyecto estudio longitudinal del desarrollo de la conducta agresiva en niños y su relación con el establecimiento de conducta antisocial en la adolescencia. Revista Mexicana de Análisis de la Conducta, 26(1) 65-89.

Beato F. L., Rodríguez, C. T., Belmonte, L. A. \& Martínez, D. C. (2004). Risk factor for eating disorders in adolescents: A Spanish community based longitudinal study. European Child Adolescent Psychiatry, 13, 287-294.

Cook-Darzens, S., Doyen, C., Falissard, B. \& Mouren, M. C. (2005). Self-perceived family functioning in 40 French families of anorexic adolescents: Implications for therapy. European Eating Disorders Review, 13, 223-226.

Dancyger, I., Fornari, V., Scionti, L. Wisotsky, W. \& Sunday, S. (2005). Do daughters with eating disorders agree with their parents' perception of family functioning? Comprehensive Psychiatry, 46, 135-139.

Davis, C., Shuster, B., Blackmore, E. \& Fox, J. (2004). Looking Good- Focus family on appearance and the risk for eating disorders. International Journal of Eating Disorders, 35, 136-144.

Emmanuelli F., Ostuzzi R., Cuzzolaro M., Baggio F., Lask B. \& Waller G. (2004). Funcionamiento familiar en adolescentes con anorexia nerviosa: una comparación de la percepción de los miembros familiares. Eating Weight Disorders, 9(1), 1-6.

Fonseca, H., Ireland, M. \& Resnick, M. D. (2002). Familial correlates of extreme weight control behaviors among adolescents. International Journal Eating Disorders, 32, 441-448.

Garner D. M. \& Garfinkel P. E. (1979). The eating attitudes test: An index of the symptoms of anorexia nerviosa. Psychological Medicine, 9, 273-279.

Garner, D. M., Garfinkel P. E. \& O'Shaughnessy, M. (1985). The validity of the distinction between bulimia with and without anorexia nerviosa. American Journal of Psychiatry, 142, 581-587.

Holston J. I. \& Cashwell C. S. (2000). Family functioning and eating disorders among collage women: A model of prediction. Journal of College Counseling, 3, 5-16.

Huon, G. F., Gunewardene, A. Hayne, A. Sankey, M., Lim, J., Piira, T. \& Walton, C. (2002). Empirical support for a model of dieting: Findings from structural equations modelling. International Journal of Eating Disorders, 31, 210-219.

Jones, C. J., Leug, N. \& Harris, G. (2006). Fatherdaughter relationship and eating psychopathology: The mediating role of core beliefs. British Journal of Clinical Psychology, 45, 319-330.

Kustlesic. V., Williamson D. A., Gleaves D. H., Barbin J. M. \& Murphy-Eberenz, K. P. (1988). The review for the diagnosis of eating disorders-IV: Application to DSM-IV diagnostic criteria. Psychological Assessment, 10(1), 41-48.

Kyriacou, O., Treasure, J. \& Schmidt, U. (2008). Expressed emotion in eating disorders assessed via self-report: An examination of factors associated with expressed emotion in careers of people with anorexia nervosa in comparison to control families. International Journal of Eating Disorders, 41(1), 37-46.

Machado, P. P., Machado, B. C., Goncalvez, S. \& Hoek, H. (2007). The prevalence of eating disorders not 
otherwise specified. International Journal of Eating Disorders, 40(3), 212-217.

Mitchell, J. E., Crosby, R. D., Wonderlich, S. A., Hill, L., le Grange, Powers, P. \& Eddy, K. (2007). Latent profile analysis of a cohort of patients with eating disorders not otherwise specified. International Journal of Eating Disorders, 40, S95-S98.

Moos, R. \& Moos, B. (1980). Family Environment Scale. Palo Alto, CA: Consulting Psychologists Press.

Okon, D. M., Greene, A. L. \& Ellen, S. J. (2003). Family interactions predict intraindividual symptom variation for adolescents with bulimia. International Journal Eating Disorders, 34, 450-457.

Sánchez C. E., Serna R. C., Seoana P. G. \& Páramo F. F. (2003). Percepción del funcionamiento familiar en familias con un miembro con trastornos del comportamiento alimentario. Revista Thomson Psicología, 1 (2), 177-188.

Seisdedos, N., Cruz, V. \& Cordero, A. (1984). Escala de Clima Social en la Familia, Madrid, TEA.

Shisslak, C. M., Mckeon, R. T. \& Crago, M. (1990). Family dysfunction in normal weigth bulimic anorexic families. Journal and Clinical Psychology, 46(2), 185-189.

Smith, M. C. \& Thelen, M. H. (1984). Development and validation of a test for bulimia. Journal of Consulting and Clinical Psychology, 52 (5), 863-872.

Strober, M. \& Humphrey, L. L. (1987). Familial contributions to the etiology and course of anorexia nerviosa and bulimia. Journal of Consulting and Clinical Psychology, 55, 634-659.

Tereno, S., Soares, I., Martins, C., Celani, M. \& Sampaio, D. (2008). Attachment styles, memories of parental rearing and therapeutic bond: A study with eating disordered patients, their parents and therapists. European Eating Disorders Review, 16, 49-58.

Treasure, J., Sepúlveda, A. R., Whitaker, W., Todd, G., López, C. \& Whitney, J. (2007). Collaborative between professionals and non-professionals in the management of eating disorders: A description of workshops focused on workshops focused on interpersonal maintaining factors. European Eating Disorders Review, 15, 24-34.

Vázquez, R., Álvarez, G. L., Mancilla, J. M. \& Raich, R. M. (2000). Dinámica familiar y actitudes alimentarias de familias con pacientes de trastorno alimentario. Psicoterapia y familia, 13(1), 33-46.

Vázquez, R., López, X., Álvarez, G. L., Franco K. \& Mancilla, J. M. (2004). Presencia de trastornos del comportamiento alimentario en hombres y mujeres mexicanas; algunos factores asociados. Psicología Conductual, 12(3), 415-427.

Vázquez, A. R. \& Raich, E. R. M. (1998). Dinámica familiar y actitudes paternas hacia los modelos estéticos corporales en familias con trastorno alimentario. Psicología y Ciencia Social, 21 (1), 59-66.

Vázquez, A. R., Raich, E. R. M., Viladrich, S. M. C., Álvarez, R. G. L. \& Mancilla, D. J. M. (2001). Tres aspectos de la vida familiar asociados a los trastornos alimentarios. Revista Mexicana de Psicología, 18(3), 325-335.

Vidović V., Jureŝa, Begovaci, I. Mahnik, M. \& Tocilj, G. (2005). Perceived family cohesion, adaptability and communication in Eating Disorders. European Eating Disorders Review, 13, 19-28.

Wade, T. D. (2007). A retrospective comparison of purging type disorders: Eating disorder not otherwise specified and bulimia nervosa. International Journal of Eating Disorders, 40 (1), 1-6.

Williamson, D. A. (1990). Assessment of eating disorders: Obesity, anorexia and bulimia nervosa. New York: Pergamon Press.

Win, S., Perkins, S., Walwyn, R., Schmidt, U., Eisler, I., Treasure, J. et al. (2007). Predictors of mental health problems and negative caregiving experiences in carers of adolescents with bulimia nervosa. International Journal of Eating Disorders, 40(2), 171-178. 